\title{
How does the U-shaped potential close above the acceleration region? A study using Polar data
}

\author{
P. Janhunen ${ }^{1}$, A. Olsson ${ }^{2,1}$, F. S. Mozer ${ }^{3}$ and H. Laakso ${ }^{1}$ \\ ${ }^{1}$ Finnish Meteorological Institute, Geophysical Research, Helsinki, Finland \\ ${ }^{2}$ Swedish Institute of Space Physics, Uppsala Division, Uppsala, Sweden \\ ${ }^{3}$ Space Sciences Laboratory, University of California, Berkeley, USA
}

Received: 28 September 1998 / Revised: 16 February 1999 / Accepted: 13 April 1999

\begin{abstract}
We present a statistical study of Polar electric field observations using auroral oval passes over Scandinavia above the acceleration region. We are especially interested in seeing whether we can find large perpendicular electric fields associated with an upward extended classical U-shaped potential drop for these passes, during which Polar is in the northern hemisphere usually at about $4 R_{E}$ altitude. We also use Polar magnetic field data to infer the existence of a field-aligned current (FAC) and conjugate ground-based magnetometers (the IMAGE magnetometer network) to check whether the event is substorm-related or not. We find several events with a FAC but only weak perpendicular electric fields at Polar. In those rare cases where the Polar electric field was large, its direction was mostly found to be incompatible with the U-shaped potential model, or it was associated with disturbed conditions (substorms), where one cannot easily distinguish between inductive and static perpendicular electric fields. We found only two cases which are compatible with the upward extended U-shaped potential picture, and even in those cases the potential value is quite small $(1-2 \mathrm{kV})$. To check the validity of the analysis method we also estimate the perpendicular electric field on the southern hemisphere, where Polar flies within or below the acceleration region, and we found a large number of inverted-V-type signatures as expected from previous studies. To explain the lack of perpendicular electric fields at high altitudes we suggest an O-shaped potential model instead of the U-shaped one.
\end{abstract}

Key words. Ionosphere (particle acceleration) · Magnetospheric physics (auroral phenomena; magnetosphere-ionosphere interactions)

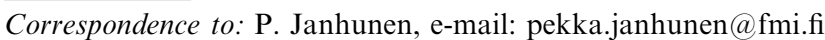

\section{Introduction}

Due to low-orbiting satellite observations (Mcllwain, 1960; Shelley et al., 1976), there is compelling evidence of the existence of upward directed parallel electric fields above auroral arcs, which was theoretically postulated a long time ago (Alfvén, 1958). However, the existence of a parallel electric field on certain field lines does not directly tell us about the geometry of the electric field in the transverse direction. Usually one adopts the "Ushaped" potential model (Fig. 1; Carlqvist and Boström, 1970) as describing the geometry. The "bottom" of the U then corresponds to the region of the parallel electric field, the auroral acceleration region. If the field lines are equipotentials above the acceleration region, the upward extensions of the " $U$ " shape (which we will call upright flanks in this paper) should be seen as perpendicular electric fields above the acceleration region, presumably extending all the way to the equatorial plane of the magnetosphere and closing only on the opposite hemisphere. We use the term upward extended U-shaped potential for the model where the flanks extend to the equatorial magnetosphere along equipotential field lines.

The magnitude of the upright flanks' perpendicular electric field can be estimated if the width of the " $U$ " and the magnitude of the parallel potential drop are known. Distances scale approximately as proportional to $B^{-1 / 2}$ in dipole field. At Polar altitude (about $4 R_{E}$ ) the magnetic field is about $500 \mathrm{nT}$ whereas in the ionosphere it is $50000 \mathrm{nT}$, thus ionosphere distances must be multiplied by a factor of ten when mapped to Polar altitude. A typical inverted-V (Frank and Ackerson, 1971; Gurnett and Frank, 1973) half-width in the ionosphere is $25 \mathrm{~km}$ (Lin and Hoffman, 1979) and a typical parallel potential drop is $5 \mathrm{kV}$, which gives $5 \mathrm{kV} /$ $250 \mathrm{~km}=20 \mathrm{mV} / \mathrm{m}$. If we try to apply the upward extended U-shaped potential model to narrow arcs whose widths can be easily less than one kilometer (Borovsky, 1993), then we would expect a $5 \mathrm{kV} /$ $5 \mathrm{~km}=1 \mathrm{~V} / \mathrm{m}$ electric field at Polar altitude. 


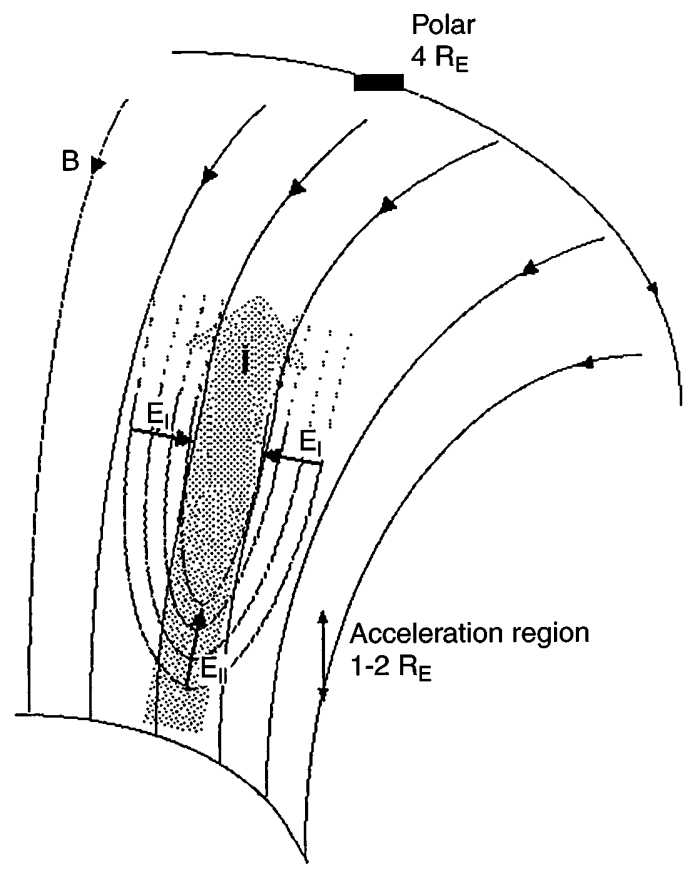

Fig. 1. The upward extended U-shaped potential model for auroral electric field. The acceleration region is usually thought to reside in the 1-2 $R_{E}$ altitude range. Polar measures the electric field above the acceleration region at about $4 R_{E}$ altitude. In the upward extended $\mathrm{U}$-shaped potential it is assumed that the flanks of " $U$ " continue upward along field lines indefinitely. We do not find support for this model in our data

Studying this question has previously been difficult since before Polar, only DE-1 and ISEE-1 have been probing the right altitude range $\left(3-5 R_{E}\right.$ above the auroral oval). In the DE-1 published results of perpendicular electric fields (Weimer et al., 1985), altitudes within and above the acceleration region were put in the same statistics. In the ISEE-1 case, the orbit was such that the passes were nearly tangential to the auroral oval, making estimation of potential drops associated with inverted-Vs difficult. Furthermore, the orbit was such that these passes occurred only when the dipole tilt angle was suitable (Mälkki and Pedersen, 1996).

In this paper we study whether the upright flanks can be seen in Polar satellite electric field measurements at $25000-35000 \mathrm{~km}$ altitude, i.e. whether perpendicular electric fields of sufficient magnitude are observed when Polar passes over the northern auroral oval. For comparison we also confirm that we find the usual signatures of inverted- $\mathrm{V}$ regions in southern hemisphere low altitude passes.

\section{Instrumentation and data analysis}

The Polar satellite was launched on 24 February 1996, in a polar orbit with $9 R_{E}$ apogee and $2 R_{E}$ perigee (Fig. 2). Polar crosses the northern auroral oval at about $4 R_{E}$ altitude. On the southern oval it is at about $1 R_{E}$ altitude, which is within or below the acceleration region. In this study we will use data from the electric

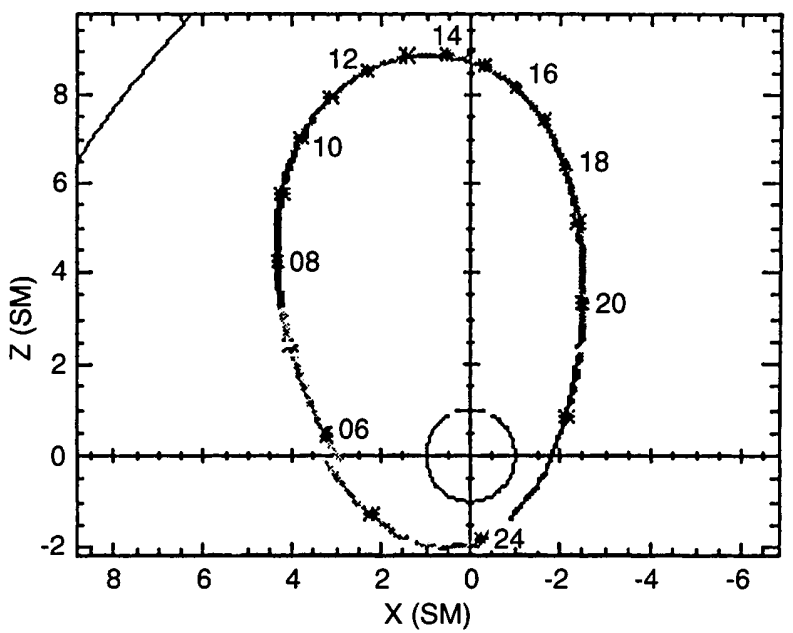

Fig. 2. Example orbit of the Polar satellite. See http://wwwspof.gsfc.nasa.gov/istp/polar/

field instrument (EFI; Harvey et al., 1995) and magnetic field experiment (MFE; Russell et al., 1995).

\subsection{Analysis of Polar EFI data}

We use the EXY and EZ spin plane electric field components of the key parameter data files; for details of the electric field instrument and the coordinate system, see Harvey et al., (1995). The time resolution of these data is one spin period (about $6 \mathrm{~s}$ ), and the data have been obtained by fitting a sinusoidal function to higher resolution data in each spin period. We integrated the spin-plane electric field along the satellite orbit to derive the potential. This construction gives the correct potential along the orbit in a stationary situation. This approach was used for example by Mälkki and Pedersen (1996). Before plotting the potentials, we removed a linear trend (a line which goes through the first and last data points) to make the small-scale variations more clearly visible. The linear trends may arise from slow temporal variations, for example, and are unimportant for the study.

In the calculation the electric field component perpendicular to the spin plane i.e. the E56 component, (see Harvey et al., 1995) was assumed zero. For this component, we do not have very reliable measurements but this component is likely to be small in these events because it is aligned with the arc. Furthermore, the effect of this component on the integrated potential would be anyway very small since the orbital plane and the spin plane are nearly the same. Also, the $\mathbf{v} \times \mathbf{B}$ term arising from the satellite motion does not contribute since it is perpendicular to the spacecraft velocity along which the integration is performed.

\subsection{Analysis of Polar MFE data}

We use the measured GSE components of the total magnetic field $\mathbf{B}$ as a starting point. A Tsyganenko model background $\mathbf{B}_{\text {model }}$ is assumed. For any vector $\mathbf{V}$ we denote the unit vector in the direction of $\mathbf{V}$ as $\mathbf{V}^{0}$ 
$\left(\mathbf{V}^{0} \equiv \mathbf{V} /|\mathbf{V}|\right)$. Denote $\hat{\mathbf{b}} \equiv \mathbf{B}^{0}$ the unit vector along the measured magnetic field. Since the plasma beta is probably rather small in this region (about $4 R_{E}$ altitude) we can assume that all current is field-aligned to first approximation. Using Ampere's law we obtain

$\mathbf{j}=\frac{\hat{\mathbf{b}}}{\mu_{0} S} \int_{\partial s} \mathrm{~d} \mathbf{l} \cdot \mathbf{B}_{1}$

where the integral is around a small loop and $S$ is the loop area and $\mathbf{B}_{1}$ is the magnetic field that we are using in the FAC computation. In order to proceed we have to assume a homogeneous direction $\hat{\mathbf{h}}$ (a direction in which the magnetic field does not change), which should be orthogonal to $\mathbf{B}_{\text {model }}$. In practice this will be approximately in the east-west direction (along the arc). A suitable $\hat{\mathbf{h}}$ is found by computing the average direction of $\mathbf{B}-\mathbf{B}_{\text {model }}$ during the event and taking the component which is locally perpendicular to $\hat{\mathbf{b}}$. Then we can define $\mathbf{B}_{1} \equiv \hat{\mathbf{h}} \hat{\mathbf{h}} \cdot\left(\mathbf{B}-\mathbf{B}_{\text {model }}\right)$. The remaining component $\mathbf{B}_{2}$ in the expansion $\mathbf{B}=\mathbf{B}_{\text {model }}+\mathbf{B}_{1}+\mathbf{B}_{2}$ is the "error" component which should be small, otherwise the FAC determination by this method is not reliable. If there are temporal variations, if the current is not fieldaligned or is not sheet-like, $\mathbf{B}_{2}$ will not be small.

Now we have an estimation of $\mathbf{B}_{1}$ in two nearby points $\mathbf{r}_{1}$ and $\mathbf{r}_{2}$, with $\Delta \mathbf{r} \equiv \mathbf{r}_{2}-\mathbf{r}_{1}$. Let us denote the magnetic field difference by $\Delta \mathbf{B}_{1} \equiv \mathbf{B}_{1}\left(\mathbf{r}_{2}\right)-\mathbf{B}_{1}\left(\mathbf{r}_{1}\right)$. By taking the loop integral over a small parallelogram with sides $\Delta r_{\perp}$ and $\hat{\mathbf{h}}$ we obtain

$j \equiv \mathbf{j} \cdot \mathbf{b}=\frac{\mathbf{h} \cdot \Delta \mathbf{B}_{1}}{\mu_{0} \hat{\mathbf{b}} \cdot \Delta \mathbf{r} \times \hat{\mathbf{h}}}$

As a consistency check we note that $j$ is independent on the sign of the homogeneous direction unit vector $\hat{\mathbf{h}}$ as it should. The current $j$ is positive in the direction of the magnetic field. If the assumptions do not hold, Eq. (2) can sometimes give a gross overestimation of the FAC, if the orientation of the vectors is bad.

\subsection{The IMAGE magnetometer chain}

The IMAGE magnetometer chain (Lühr et al., 1998) consists of several ground-based magnetometers over northern Scandinavia. In this study we employ these stations to see whether notable magnetic signatures are associated with the Polar passes and especially whether substorm signatures are seen.

\section{Observations}

\subsection{Northern auroral oval}

We began this study by collecting all events where Polar is above northern Scandinavia. Specifically, we used the criteria that the Polar footprint must be within $\pm 6^{\circ}$ geographic latitude and within $\pm 30^{\circ}$ geographic longitude from the Kilpisjärvi magnetometer station. We limited the study to auroral passes over Scandinavia because we want to check the ground magnetograms in order to put the events into geophysical context and to reject substorm-related events from the study. As we are doing a conjunction study with a magnetometer network, we can use a relatively large conjunction window to collect enough events. In order to remove substormrelated events reliably, we consider $3 \mathrm{~h}$ of magnetometer data. If a substorm occurs on the eastern side of the window, the Earth has time to rotate within this time so that some of the magnetometers detect the substorm signals. If the substorm occurs on the western side of the window, it usually emits eastward propagating structures which propagate over the magnetometers, again causing detectable signals.

The time period of our study covers 960401-980929. Substorm-related events are excluded from the study because rapid horizontal motions of auroral arcs give rise to perpendicular electric fields which may well mask the upright flank associated electric fields that we are looking for. For example, an ionospheric speed of $5 \mathrm{~km} / \mathrm{s}$ translates to $50 \mathrm{~km} / \mathrm{s}$ at Polar altitude, corresponding to $25 \mathrm{mV} / \mathrm{m}$ perpendicular electric field, which is of the same order of magnitude as the upright flank associated fields that we are looking for.

As a first step, we classify the events according to the strength of the ground magnetic signature (B), Polar electric field (E) and Polar variation magnetic field (F). The variation field was computed by subtracting a background magnetic field, and we assume that the variation is primarily due to a field-aligned current (FAC). A "strong" ground magnetic signature is defined to be a variation in the geographic north component which exceeds $50 \mathrm{nT}$. For a "strong" Polar electric field we use the limit $20 \mathrm{mV} / \mathrm{m}$. We also integrated the electric field along the satellite orbit as explained above in section "Instrumentation and data analysis" to get the potential. Our classification scheme here is based on the electric field strength rather than the potential values, but the conclusions presented below are mainly based on a visual inspection of the potential curves together with other data. Polar magnetic field signature was considered "strong" if a clearly identifiable peak or wave structure of more than $10 \mathrm{nT}$ was seen. In all cases the classification was done manually and thus some subjectivity remains. We denote the events by combining capital and lower case letters, for example BeF means strong ground magnetic signal, weak Polar electric field and strong Polar magnetic field. Likewise, bEF means weak ground signal but strong Polar signals in both electric and magnetic fields.

The total number of events thus processed was 78 . The classification is shown in Table 1 . In addition to these northern hemisphere high altitude events, we looked at 24 southern hemisphere low altitude events. The southern hemisphere events are not included in Table 1 but are discussed separately below.

In Figs. 3 and 4 we show typical events in classes BeF and BEF, respectively. Figures 5 and 6 show two events that could be supporting the upward extended U-shaped potential. Figures 7-9 show the latitudinal, altitude and seasonal distributions of the events. We have excluded 
Table 1. Classification of events

\begin{tabular}{|c|c|c|c|c|c|c|}
\hline & IMAGE & Polar E & Polar F & Abbrev. & Number & Percentage \\
\hline \multirow[t]{3}{*}{ be } & small & small & small & bef & 43 & 55 \\
\hline & small & small & Large & beF & 16 & 21 \\
\hline & small & small & missing & be & 2 & 4 \\
\hline \multirow[t]{2}{*}{$\mathrm{Be}$} & Large & small & small & Bef & 1 & 1 \\
\hline & Large & small & Large & $\mathrm{BeF}$ & 4 & 5 \\
\hline \multirow[t]{3}{*}{$\mathrm{bE}$} & small & Large & small & $\mathrm{bEf}$ & 0 & 0 \\
\hline & small & Large & Large & $\mathrm{bEF}$ & 2 & 3 \\
\hline & small & Large & missing & $\mathrm{bE}$ & 1 & 1 \\
\hline \multirow[t]{3}{*}{$\mathrm{BE}$} & Large & Large & small & BEf & 0 & 0 \\
\hline & Large & Large & Large & $\mathrm{BEF}$ & 2 & 3 \\
\hline & $\begin{array}{l}\text { Large/ } \\
\text { Substorm }\end{array}$ & Large & Large & BsEF & 7 & 9 \\
\hline
\end{tabular}
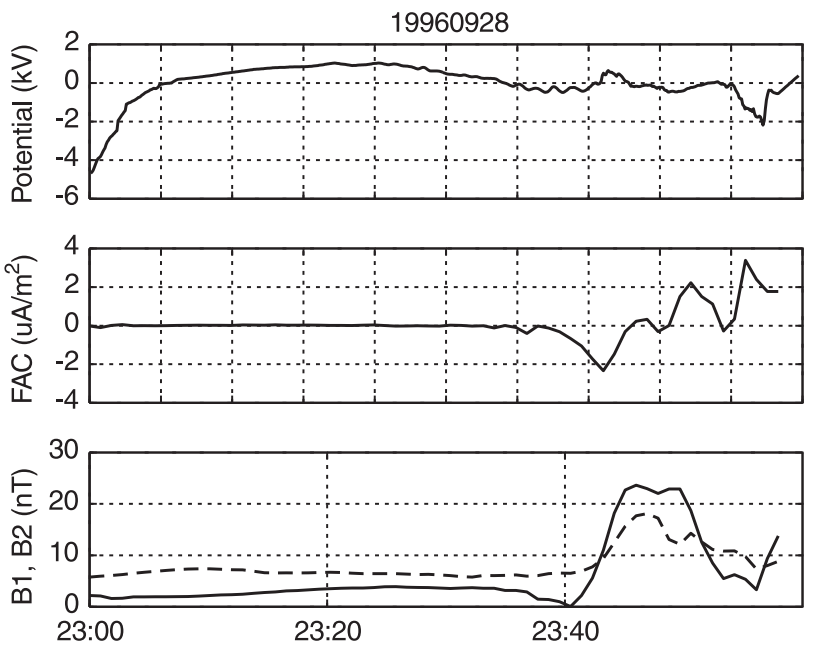

Fig. 3. Event 960928 23:35-23:45 UT (the BeF category). Since $B_{2}$ is not much smaller than $B_{1}$, the FAC estimate is not very reliable. The potential is weak and has the wrong sign to support the upward extended U-shaped potential picture
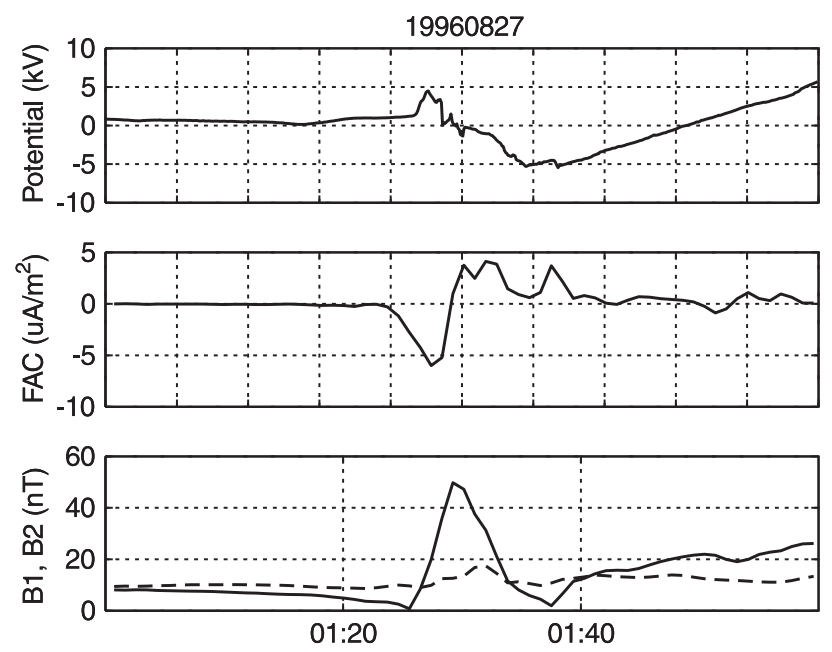

Fig. 4. Event 9608827 01:25-01:35 UT (the BEF category). Since $B_{2}$ is smaller than $B_{1}$, the FAC estimate should be rather reliable. The potential has the wrong sign to support the upward extended Ushaped potential picture
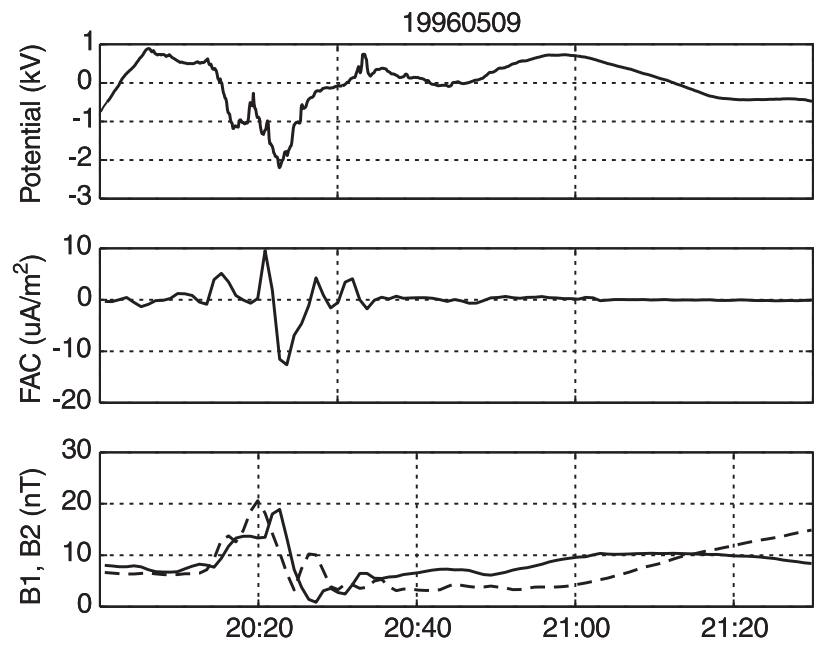

Fig. 5. Event 960509 20:15-20:30 UT. Since $B_{2} \sim B_{1}$, the FAC is not very reliable. As the potential and the FAC are both negative, this could support the upward extended U-shaped potential, but the magnitude of the potential is not very large. Among our 78 events this is one of the two that are somewhat compatible with the upward extended U-shaped potential picture
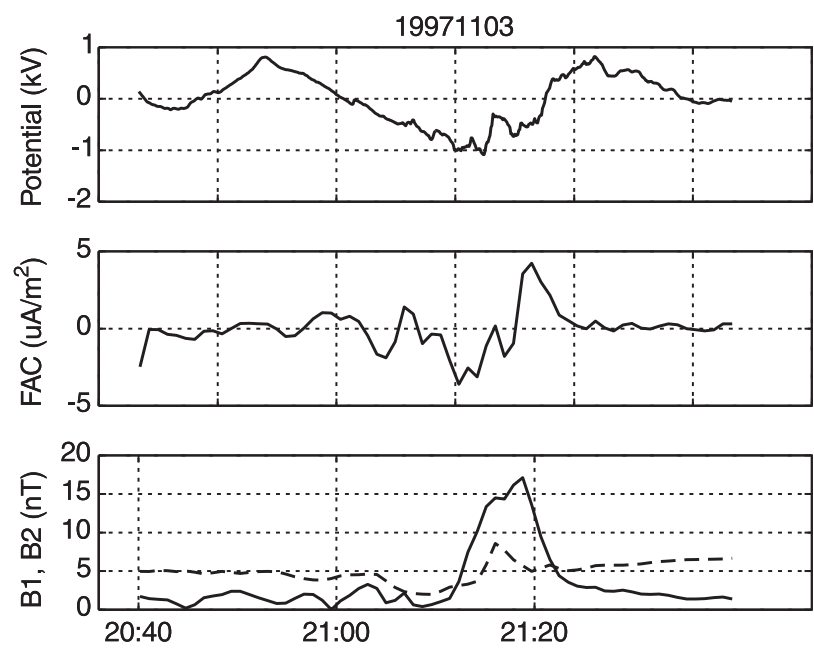

Fig. 6. Event 971103 21:05-21:25 UT. $B_{2}$ is smaller than $B_{1}$ so the FAC estimate is rather reliable. The potential and the FAC have the same sign so this event could support the upward extended U-shaped potential picture, but the magnitude of the potential $(\sim 1 \mathrm{kV})$ is small. Among our 78 events only two are somehow compatible with the upward extended U-shaped potential picture, and this is one of them

events in the 06-18 MLT range from the study. There are no clear correlations between the class of the event and mean local time, altitude or season.

\subsection{Southern auroral oval}

For comparison and testing our analysis methods, we took 24 southern hemisphere events where Polar is within or even below the acceleration region. Other than being on the southern hemisphere, we process these 


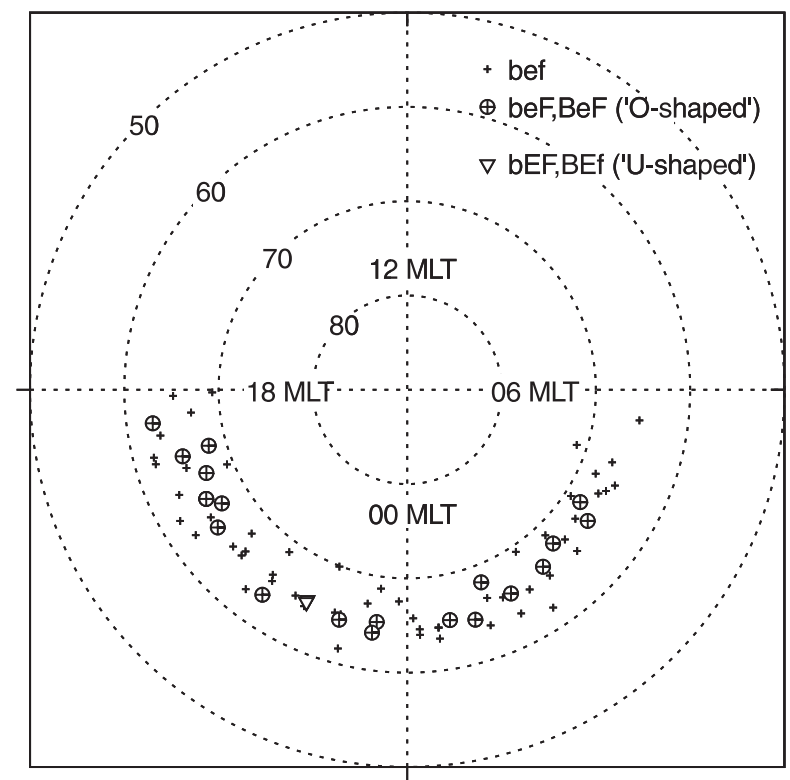

Fig. 7. The MLT and magnetic latitude distribution of the events. Weak events are marked with \pm , events with magnetic field signatures but weak electric fields by $\oplus$, and events where the electric field is strong by a triangle. There are two triangles in the plot which happen to overlap each other

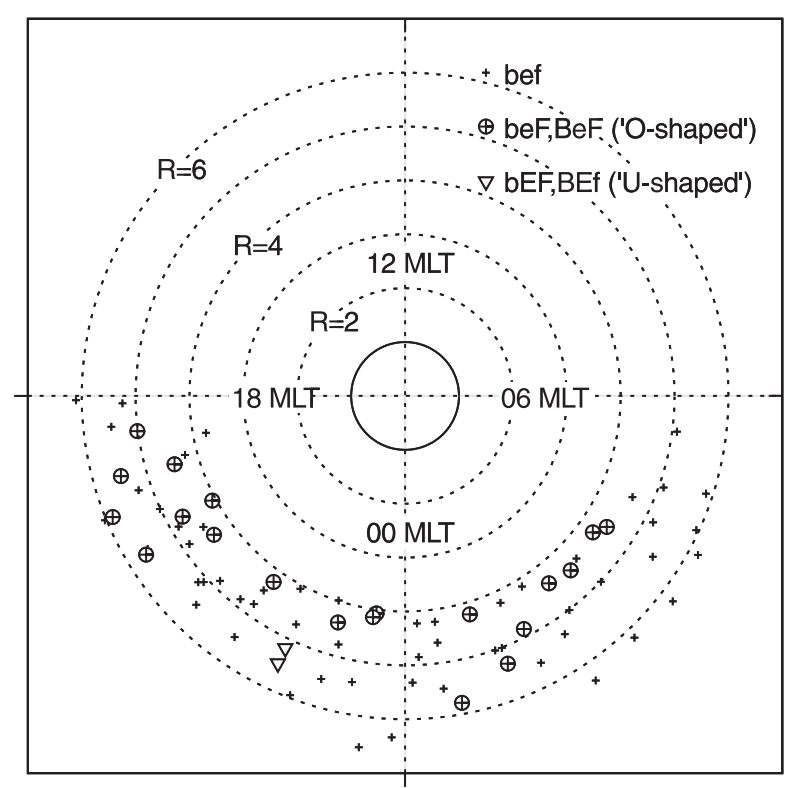

Fig. 8. Same as Fig. 7, but the distance from the center of the figure now gives the radial distance from the Earth in units of Earth radius

events in the same way as the northern hemisphere events. Among these events we find four cases of negative potential of magnitude $5-15 \mathrm{kV}$ associated with clear indication of upward field-aligned current of $0.7-2.7 \mu \mathrm{A} \mathrm{m} \mathrm{m}^{-2}$ magnitude as estimated from the Polar magnetometer. This is in accordance with the classical view of acceleration region associated with inverted-V precipitation.

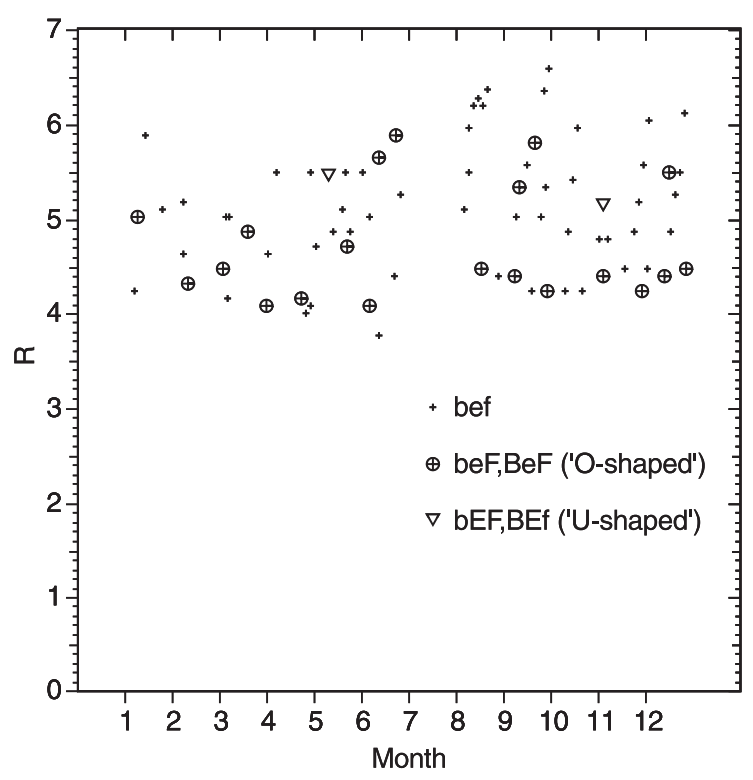

Fig. 9. Same as Fig. 7, showing the seasonal distribution of the events

Thus, on low-altitude Polar passes, our frequency of finding convergent electric field signatures with upward field-aligned current is high, about $17 \%$. Indeed, it has been established earlier (Lin and Hoffman, 1979) that inverted-V events are very common. Since the low-altitude Polar passes may be sometimes below the acceleration region, we expect that the real frequency of seeing convergent electric fields is even higher than $17 \%$. Thus, we should expect at least $17 \%$ of the northern hemisphere passes to have similar potential drop and field-aligned current signatures, if the upward extended U-shaped potential picture was correct.

\section{Discussion}

We go through each category in Table 1, discussing the physical implications in each case.

Category bef $(55 \%)$. All quantities are small and thus the bef events are not interesting for our study. This category is nevertheless the most common one.

Category beF $(21 \%)$. There must be some FAC (and, probably, some precipitation) since there is a Polar magnetic field signal. To explain the absence of a ground magnetic structure, this FAC should flow as an adjacent pair of upward and downward currents. The absence of an electric field while a FAC is there cannot be explained by the upward extended U-shaped potential model. This is the most common type of event with some activity in at least one of the variables (E, B or F).

Category bEf $(0 \%)$. In this case there is no FAC and no ground magnetic signature, but still an electric field at Polar. We found no events in this category.

Category bEF (3\%), two events. Having both electric field and FAC signatures at Polar would give support to the upward extended U-shaped potential picture if the 
directions of the vectors also match. We show the two events (960509 and 971103) in Figs. 5 and 6. In 960509 the determination of the field-aligned current is rather poor $\left(B_{2} \sim B_{1}\right)$, but the magnetic field signature could correspond to a strong $\left(10 \mu \mathrm{A} \mathrm{m}^{-2}\right)$ negative (upward) field-aligned current with a $2 \mathrm{kV}$ minimum in potential. In 971103 the determination of the field-aligned current is more reliable and both FAC and potential have a bipolar signature. However, in both these cases the potentials are small $(1-2 \mathrm{kV})$ compared to what we typically found for low altitudes in this study $(5-15 \mathrm{kV})$ or what has been found in earlier studies (Lin and Hoffman, 1979; Olsson et al., 1996, 1998).

Category Bef $(1 \%)$. In this case Polar gives no signal in either electric or magnetic fields, but the ground magnetometers do. The ground magnetic perturbations would be caused by a time-varying electrojet current, for instance. We found only one event in this category and its ground perturbation was close to the limit, not much larger than $50 \mathrm{nT}$.

Category BeF $(5 \%)$. Having a FAC and ground magnetic perturbation without Polar electric field cannot be explained by the upward extended U-shaped potential model.

Category BEf $(0 \%)$. In this case we have a ground magnetic signature and Polar electric field but no sign of FAC at Polar. We have no events in this category.

Category BEF (3\%). Having a FAC, ground magnetic field and Polar electric field signatures would support the upward extended U-shaped potential picture if the directions of the vectors were also correct. We have two events in this category $(960827,971017)$, and in both cases the direction of the Polar electric field is incompatible with the upward extended U-shaped potential. Thus, the Polar electric field must be caused by something else than the flanks of an upward extended U-shaped potential in these cases. Such mechanism could be time-varying phenomena such as Alfvén waves, for instance.

Category BsEF (9\%). These events differ from BEF events in that one can identify a substorm in the ground magnetograms. As substorms are usually associated with rapid horizontal motion of the auroral structures, one would expect large perpendicular electric fields at Polar altitude also arise because of this. These events are thus excluded from further study.

Based on our data we see that there are not many events, only 12 out of 78, with large electric fields (larger than $20 \mathrm{mV} / \mathrm{m}$ ), and in these cases the event is either substorm-related or the electric field direction is mostly not compatible with the upward extended U-shaped potential picture. This raises doubts about the applicability of the upward extended U-shaped potential picture to explain these results at all. The distribution of events, especially category beF, suggests that large low-altitude potential structures must be closed below the Polar altitude $\left(\sim 4 R_{E}\right)$. This will imply that below Polar there must be some downward parallel electric fields. Then, instead of a U-shaped potential structure, we consider an "O-shaped" potential (Fig. 10), i.e. the possibility that there is an downward directed parallel

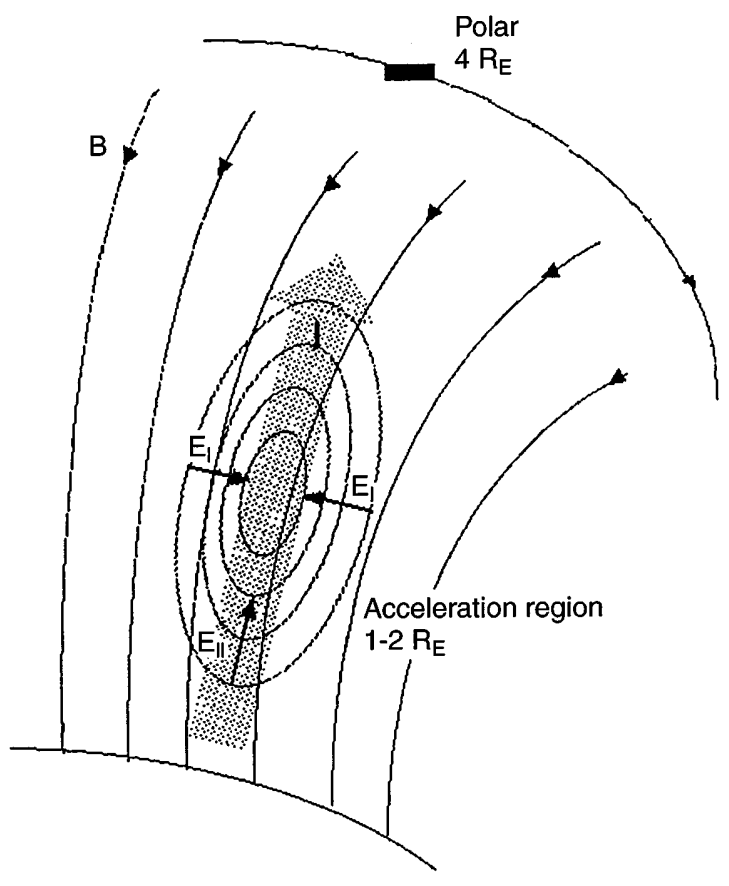

Fig. 10. The O-shaped potential model for auroral electric field. Our data support the O-shaped model rather than the upward extended U-shaped model displayed in Fig. 1. In the O-shaped potential model there need not be any strong electric fields at $4 R_{E}$ Polar altitude even though simultaneous low-altitude observations would show electrostatic shocks and parallel potential drops. We found support for this configuration in $26 \%$ of cases

electric field above the acceleration region. Thus, the equipotentials would be closed loops and we would expect no signature at Polar altitude of the upward flanks of the upward extended U-shaped potential.

One could also think of combinations between $\mathrm{U}$ and O shaped potentials, for example, a partly closing Oshaped potential. It is interesting that the electric field has sometimes a divergent (e.g., see Figs. 3 and 4) rather than convergent nature within an upward FAC region, speaking in favour of a slightly "over-closed" O-shaped potential (Fig. 11) rather than a combination of $\mathrm{O}$ and $\mathrm{U}$.

The next step in the investigation of the applicability of O-shaped and upward extended U-shaped potential drops would be to study some of the individual events more carefully and to find more events where Polar and some low-orbiting satellite are in conjunction. In this way one could get an independent estimate of the parallel potential drop, as well as the width of the inverted- $\mathrm{V}$ region and field-aligned current, from the low-orbiting satellite. It would also be of importance to find events where ground-based all-sky cameras are in conjunction with Polar and to study theoretically possible mechanisms leading to $\mathrm{O}$-shaped potentials in upward field-aligned current regions.

The existence of an upward parallel electric field corresponding to a several $\mathrm{keV}$ potential drop above many inverted- $\mathrm{V}$ arcs has been demonstrated using lowaltitude spacecraft quite convincingly. It has also been demonstrated that the integration of the electric field along the orbit of a spacecraft moving through an 


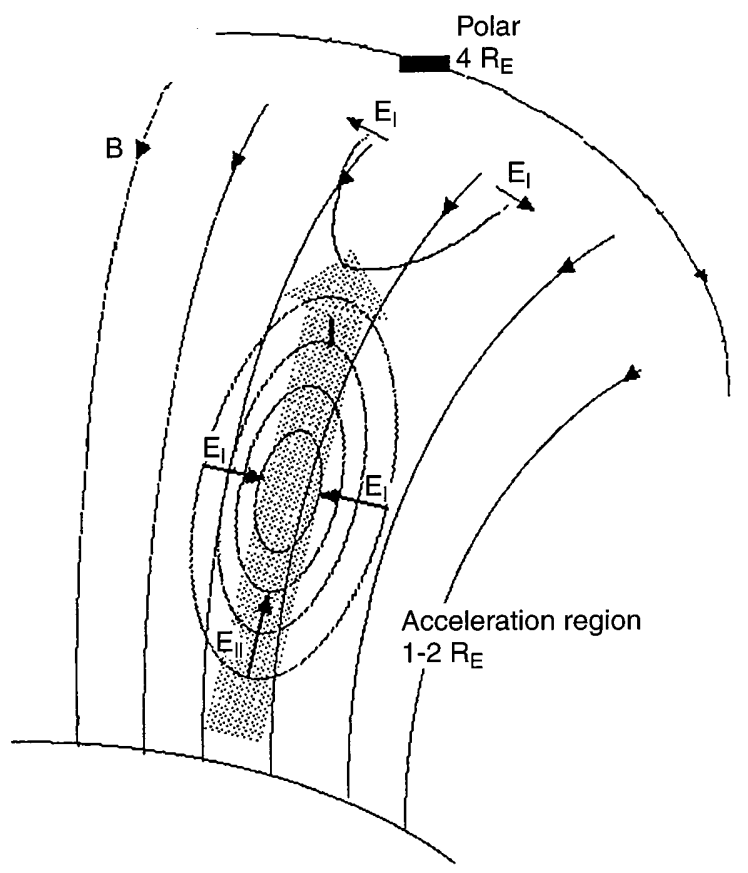

Fig. 11. An "over-closed" O-shaped potential. The downward parallel electric field on top of the "O" is slightly larger than the upward field on the bottom, resulting in divergent perpendicular electric field at Polar altitude. This kind of configuration is suggested by some of our events

upward ion beam region yields the same energy as the ion beam energy (McFadden et al., 1998), which not only supports the existence of a parallel electric field below the spacecraft but also supports the U-shaped potential picture in the region below about $4000 \mathrm{~km}$ altitude. If one assumes a static electric field, the Oshaped potential geometry is the only way to have an agreement between these results and ours. The other possibility would be to have an inductive electric field, to which the topological constraints present in the static potential model do not apply. It is not clear, however, how such an inherently time-dependent model could explain stable auroral arcs.

\section{Summary}

We summarize our findings briefly.

1. Electric fields that were large enough to conform with the upward extended U-shaped potential drop model are relatively rare in Polar altitude $(15 \%$ of all events; the total number of events is 78). Cases where the electric field was large were usually ( 7 out of 12 ) related to substorms. As these events can be associated with large convective electric fields related to horizontal motion, we exclude these events from further consideration in this study. Of the remaining five events, three have their electric field directions inconsistent with an upward extended U-shaped potential. The large electric fields in these events must thus be explained by time-dependent phenomena such as Alfvén waves. The remaining two cases (Figs. 5 and 6) are, in principle, compatible with the upward extended U-shaped potential, but the magnitude of the potential is fairly low (below a few $\mathrm{kV}$ ) compared with the intensity of the field-aligned current.

2. Within our events, we find no correlations between the character of the events and altitude, mean local time or season.

3. A total of $26 \%$ of the cases have a signature of FAC but no electric field at Polar (i.e. they belong to category beF or BeF). These cases can not be explained by the upward extended U-shaped potential model, but are compatible with the O-shaped model.

4. In southern hemisphere low-altitude Polar passes we find cases ( $17 \%$ of 24 cases) having a convergent perpendicular electric field and an upward field-aligned current. This finding is in agreement with earlier studies and indicates that there is no serious error in our analysis method.

5. As the percentage of seeing convergent electric fields was $17 \%$ at low altitude, it is not reasonable to think that all our 78 high altitude events have by chance missed the upward extended U-potentials. On the contrary we found $26 \%$ of the high altitude events seeing field-aligned currents without significant electric fields.

6. As an alternative to the U-shaped model we propose an $\mathrm{O}$-shaped potential model. In this model there is a downward parallel electric field region above the acceleration region, and no net potential drop between the ionosphere and the magnetosphere. Another possibility would be to assume that inductive electric fields play an important role.

Acknowledgements. We thank C.T. Russell for providing us with the Polar magnetic field data. The IMAGE magnetometer network is supported by FMI of Finland and we would like to thank Ari Viljanen for helping us analyzing it. We thank Per-Arne Lindqvist of $\mathrm{KTH} /$ Stockholm for useful discussions about electric field data. The work of AO was partly supported by the Knut and Alice Wallenberg Foundation. Work at the Univ. California Berkeley was performed under NASA contracts NAS 5-30367 and NAG 5-3182.

Topical Editor K.-H. Glassmeier thanks N.C. Maynard and J.E. Borovsky for their help in evaluating this paper.

\section{References}

Alfvén, H., On the theory of magnetic storms and aurora, Tellus, 10, 104, 1958

Borovsky, J. E., Auroral arc thickness as predicted by various theories, J. Geophys. Res., 98, 6101-6138, 1993.

Carlqvist, P., and R. Boström, Space-charge regions above the aurora, J. Geophys. Res., 75, 7140-7146, 1970.

Frank, L. A., and K. L. Ackerson, Observations of charge particle precipitation into the auroral zone, J. Geophys. Res., 76, 36123642, 1971.

Gurnett, D. A., and L. A. Frank, observed relationship between electric field and auroral particle precipitation, J. Geophys. Res., 78, 145-170, 1973.

Harvey, P., F. S. Mozer, D. Pankow, J. Wygant, N. C. Maynard, H. Singer, W. Sullivan, P. B. Anderson, R. Pfaff, T. Aggson, A. Pedersen, C. G. Falthammar and P. Tanskanen, The electric field instrument on the polar satellite, Space Sci Rev., 71, 583596, 1995.

Lin, C. S., and R. A. Hoffman, Characteristics of the inverted-V event, J. Geophys. Res., 84, 1514-1525, 1979. 
Lühr, H., A. Aylward, S. C. Bucher, A. Pajunpää, K. Pajunpää, T. Holmboe, and S. M. Zalewski, Westward moving dynamic substorm features observed with the IMAGE magnetometer network and other ground-based instruments, Ann. Geophys., 16, 425-440, 1998.

Mälkki, A., and A. Pedersen, Mapping of ISEE-1 auroral electric fields to the ionosphere and to the equatorial magnetosphere, J. Geomag. Geoelectr., 48, 897-913, 1996.

McFadden, J. P., C. W. Carlson, R. E. Ergun, F. S. Mozer, M. Temerin, W. Peria, D. M. Klumpar, E. G. Shelley, W. K. Peterson, E. Moebius, L. Kistler, R. Elphic, R. Strangeway, C. Cattell, and R. Pfaff, Spatial structure and gradients of ion beams observed by FAST, Geophys. Res. Lett. 25, 2021-2024, 1998.

McIlwain, C. E., Direct measurement of particles producing visible aurora, J. Geophys. Res., 65, 2727, 1960.
Olsson, A., A. I. Eriksson, and P. Janhunen, On the current-voltage relationship in auroral breakups and westward traveling surges, Ann. Geophys., 14, 1265-1273, 1996.

Olsson, A., L. Andersson, A. I. Eriksson, J. Clemmons, R. E. Erlandsson, G. Reeves, T. Hughes, and J. S. Murphree, Freja studies on the current-voltage relation in substorm related events, J. Geophys. Res., 103, 4285-4301, 1998.

Russell, C. T., R. C. Snare, J. D. Means, D. Pierce, D. Dearborn, M. Larson, G. Barr, and G. Le, The GGS/Polar Magnetic Fields Investigation, Space Sci. Rev., 71, 563-582, 1995.

Shelley, E. G., R. G. Johnson, and R. D. Sharp, Satellite observations of an ionospheric acceleration mechanism, Geophys. Res. Lett., 3, 654-656, 1976.

Weimer, D. R., C. K. Goertz, D. A. Gurnett, N. C. Maynard, and J. L. Burch, Auroral zone electric fields from DE 1 and 2 at magnetic conjunctions, J. Geophys. Res., 90, 7479-7494, 1985. 